See discussions, stats, and author profiles for this publication at: https://www.researchgate.net/publication/39662782

\title{
Access to spectrally resolved ultra-dense hot low z emissivities and opacities
}

Article in AIP Conference Proceedings · January 2002

Source: OAI

CITATIONS

READS

28

15 authors, including:

Paulo Angelo

Sorbonne Université

104 PUBLICATIONS 274 CITATIONS

SEE PROFILE

Elisabeth Dalimier

Sorbonne Université-Faculty of Science and Engineering

213 PUBLICATIONS 660 CITATIONS

SEE PROFILE

Some of the authors of this publication are also working on these related projects:

Project DEMO european View project

Project DEMO EUROFusion View project

Emmanuelle Dufour

Atomic Energy and Alternative Energies Commission

38 PUBLICATIONS 213 CITATIONS

SEE PROFILE

Patrick Sauvan

National Distance Education University

147 PUBLICATIONS 466 CITATIONS

SEE PROFILE 


\section{AIP $\mid$ Proceedings}

\section{Access to Spectrally Resolved UltraDense Hot Low Z Emissivities and Opacities}

R. Schott, F. Philippe, P. Angelo, E. Dufour, A. Poquérusse, E. LeboucherDalimier, P. Sauvan, P. Velarde, F. Ogando, E. Minguez, J. M. Gil, J. G. Rubiano, R. Rodriguez, P. Martel, and R. Mancini

Citation: AIP Conference Proceedings 645, 340 (2002); doi: 10.1063/1.1525474

View online: http://dx.doi.org/10.1063/1.1525474

View Table of Contents: http://scitation.aip.org/content/aip/proceeding/aipcp/645?ver=pdfcov

Published by the AIP Publishing

\section{Articles you may be interested in}

Ultradense Hot Low Z Line Transition Opacity Simulations

AIP Conf. Proc. 645, 352 (2002); 10.1063/1.1525475

Comparative study of time-resolved K-shell spectra from aluminum plasmas generated by ultrashort laser pulses at 395 and $790 \mathrm{~nm}$

Appl. Phys. Lett. 80, 198 (2002); 10.1063/1.1432106

Overcritical density plasma diagnosis inside laser-produced craters

Appl. Phys. Lett. 79, 177 (2001); 10.1063/1.1381413

Ionic correlation effects on opacity and emissivity of ultra-dense hot low $Z$ plasmas

AIP Conf. Proc. 559, 39 (2001); 10.1063/1.1370584

X-ray emission from metallic $(\mathrm{Al})$ and dielectric (glass) targets irradiated by intense ultrashort laser pulses Appl. Phys. Lett. 73, 1334 (1998); 10.1063/1.121887 


\title{
Access to Spectrally Resolved Ultra-Dense Hot Low Z Emissivities and Opacities
}

\author{
R. Schott ${ }^{1}$, F. Philippe ${ }^{1}$, P. Angelo ${ }^{1}$, E. Dufour ${ }^{1}$, A. Poquérusse ${ }^{1}$, E.

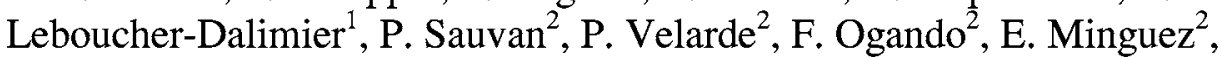

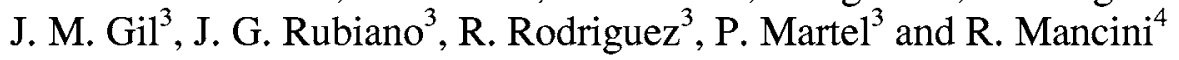 \\ ${ }^{1}$ Physique Atomique dans les Plasmas Denses-LULI, CNRS-CEA-Ecole Polytechnique-Université Paris \\ 6, 75252 Paris cedex 05 and 91128 Palaiseau cedex. \\ ${ }^{2}$ Instituto de Fusion Nuclear, E.T.S. de Ingenieros Industriales U.P.M., 28006 Madrid, Spain. \\ ${ }^{3}$ Departamento de Fisica, Universidad de Las Palmas de Gran Canaria, Spain. \\ ${ }^{4}$ Physics Department University of Nevada, Reno, NV 89557, USA
}

\begin{abstract}
We present here experimental studies of broadened bound-bound emissivities and opacities of low $\mathrm{Z}$ plasmas that are the best candidates for exhibiting ion and electron correlations. First we report on an emission experiment where a new target design is used to access the highest densities. Such targets irradiated by an intense long laser pulse generate plasmas well adapted to model and extract opacities. The measurements are compared to theoretical results obtained from simulations involving new atomic/molecular physics models that take into account detailed line profiles. We end up with the description of an absorption experiment in progress, this experiment using the same targets.
\end{abstract}

\section{INTRODUCTION}

The present work is devoted to measurements of bound-bound opacities of lasershocked dense aluminum plasmas by emission and absorption spectroscopy. For those plasma conditions corresponding to astrophysical situations, the white dwarf atmosphere, the center of the sun, there is a lack of information, computations and measurements. The study is then decisive for the knowledge of the radiative transfer and the optimization of the X-ray sources. With the access to the new generation of large-scale facilities and the intense lasers, it is now possible to reach partly, in laboratories, the temperature-density domain $\left[10^{5} \mathrm{~K}-1 \mathrm{~g} . \mathrm{cm}^{-3} ; 10^{6} \mathrm{~K}-10^{2} \mathrm{~g} . \mathrm{cm}^{-3}\right]$. The study of such extreme conditions for low $\mathrm{Z}$ plasmas is extremely interesting for the new atomic/molecular physics involved and for the crucial role of the line broadening [1]. Because of the low $\mathrm{Z}$ and the high density the strong ionic correlations act through the Stark effect in the spectral line shapes and consequently on opacities. The quasimolecular model has been proved to be adapted to this plasma regime (strong correlations, moderate coupling) for the simulations of opacities [2].

Two diagnostics, "emission spectroscopy » and "absorption spectroscopy », for the measure of bound-bound opacities are developed in this work and they are both at the limit of the feasibility: as a matter of fact the plasma is too "cold" for emission measurements and too "hot" for absorption measurements. The aim of the work is to

CP645, Spectral Line Shapes: Volume 12, $16^{\text {th }}$ ICSLS, edited by C. A. Back (c) 2002 American Institute of Physics 0-7354-0100-4/02/\$19.00 
compare both diagnostics. At first some important improvements devoted to the target design and to the spectrographs are presented.

\section{IMPROVING THE EXPERIMENTAL SETUP FOR THE ACCESS TO DENSE PLASMAS}

The experiments have been carried out at the Ecole Polytechnique, Palaiseau. The neodymium laser chain provides a 600 ps gaussian pulse with energy of $80 \mathrm{~J}$ and a wavelength of $1,053 \mu \mathrm{m}$. In the emission experiment, the initial $\omega$ beam is converted into a $4 \omega$ beam thanks to KDP crystal (figure 1). The $4 \omega$ beam (25J delivered in $500 \mathrm{ps}$ ) is focused onto a massive aluminum target, the $100 \mu \mathrm{m}$ focal spot allowing an intensity as high as $2.10^{14} \mathrm{~W} . \mathrm{cm}^{-2}$. The absorption experiment described in the last section requires a second beam at double frequency in order to irradiate a backlighter. There is no need to have a $4 \omega$ beam for that because the requirement of high density is less important than the brightness of the source in the spectral window for the aluminum absorption.

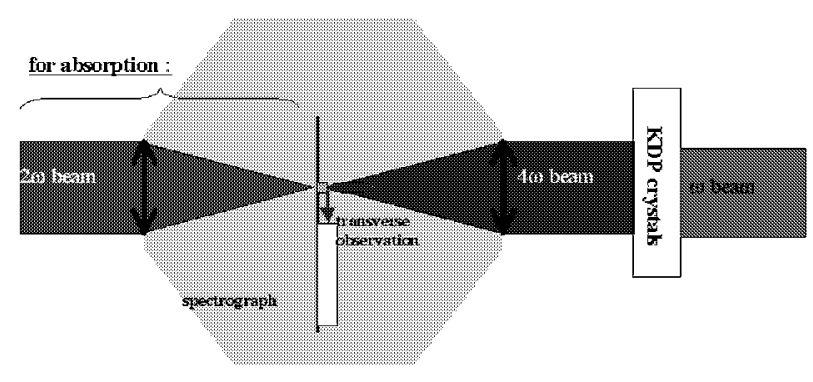

FIGURE 1. Interaction chamber schematic.

\section{The Targets}

Structured targets are used in this experiment (see figure 2). A slice of aluminum, the emissive material, is inserted in a carbon substrate. This configuration enables the aluminum plasma to be confined transversally by the carbon plasma. A second interest stems from the control of the transverse optical path through the aluminum thickness. For a thickness lower than $70 \mu \mathrm{m}$, the transverse inhomogeneity due to the laser irradiation pattern of the focal spot can be assumed to be negligible. Obviously for such targets directly coated on the spectrograph slit, the densest part of the aluminum plasma is very difficult to access in a transverse direction because it is located in the shocked region inside the crater. The first attempt to overcome this difficulty was to incline the target over the slit, allowing a quasi-transverse observation inside the crater. However, this design generates space integrations over plasma regions where 
the density is lower than the one we want to probe, and the very dense region that can be accessed transversally is rather limited.

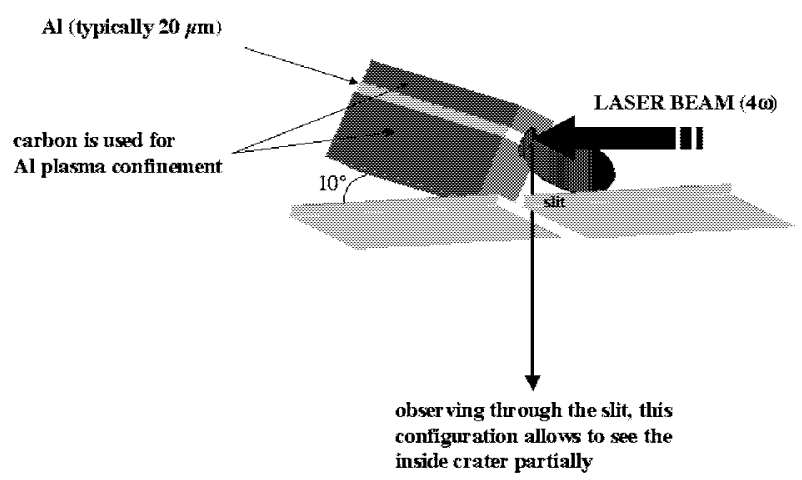

FIGURE 2. Structured target.

Recently, a second attempt to create and access ultra-dense and hot plasma has been successful. The figure 3 shows a schematic of this new design. This time, the laser hits the edge of a three layered-foil $(\mathrm{CH} / \mathrm{Al} / \mathrm{CH})$ lying on a support so that the foil sticks out the support. The total thickness of the foil must not exceed the focal spot diameter, so that the plastic is entirely converted into plasma during the laser shot. This plasma ensures the aluminum plasma confinement. The laser-target interaction gives rise to a nick instead of a crater, and this innovation is the key point for a rigorous transverse observation of the ultra-dense plasma. Moreover, using this implementation, the space resolution involves a larger part of ultra-dense plasma, and the integration over less dense regions is removed.

This newly designed target is also suitable for absorption spectroscopy. As a matter of fact, it is now possible for the back-lighter emission to go through the densest part of the aluminum plasma.

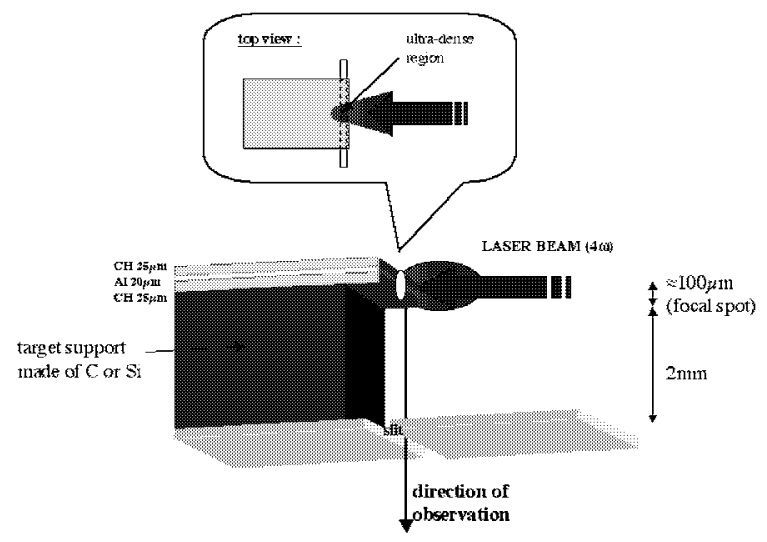

FIGURE 3. New target design. 


\section{The Spectrograph}

The reabsorbed emission is assessed with an ultra-high resolution $(\Delta \lambda / \lambda=8000)$ spectrograph (see figure 4). A PET crystal, curved in order to fit a circle whose radius is twice the Rowland circle radius, is used in Johann geometry. The spectral window includes the aluminum Lyman and helium-like lines from 5.3 to $7.4 \AA$. A beryllium window protects the DEF film from the visible light.

The aluminum plasma is very close to the slit $(2 \mathrm{~mm})$, allowing a very high space resolution with a transverse magnification of 100 . On the figure 4 , the upper edge of the slit acts as a knife and the film detects the emission with progressive space integration along the laser-target axis. For instance, the emission on the film at $0.2 \mathrm{~mm}$ from the bottom of the lines corresponds to the emissive volume of the densest part of the plasma extending on about $2 \mu \mathrm{m}$. This volume corresponds to the plasma to be probed in this work by emission or absorption spectroscopy.

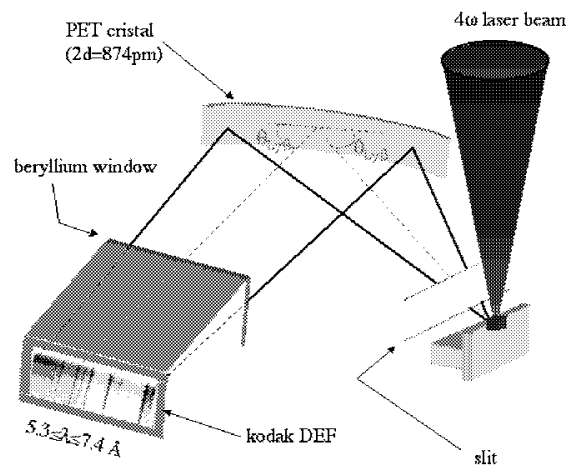

FIGURE 4. The different elements of the spectrograph.

\section{The Improvements}

An example of the detected emission from two different targets is given on the left part of figure 5. The upper film has to be related to a previous inclined target while the lower film corresponds to a newly three-layered foil target. The spectral windows and the dispersions are the same for both films. A comparison between the two films reveals that the maximum broadening of the lines is more important with the newly designed target than with the previous target. This broadening is the signature of the Stark effect, i.e. the density effect; thus denser plasma is observed with the new design. The distance, on the film, over which this consequent Stark broadening is observed gives a clue to the extension of the dense plasma region. This distance is increased with the three-layered target because the observation takes place in the nick where most of the dense region is located while the previous inclined target only allowed the probing of a small part of the crater.

The right part of figure 5 displays several scans taking into account all instrumental corrections. These scans are connected to the films on the left hand side. For both sets, space integrations over the first 4,8 and $12 \mu \mathrm{m}$ of dense plasma are performed, and 
the spectra are shifted from one to another for more clarity. The last visible Lyman

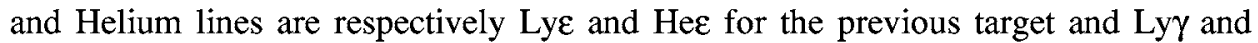
He $\gamma$ for the newly designed target. According to the Inglis-Teller limit, this statement provides a complementary argument for the access to higher densities.
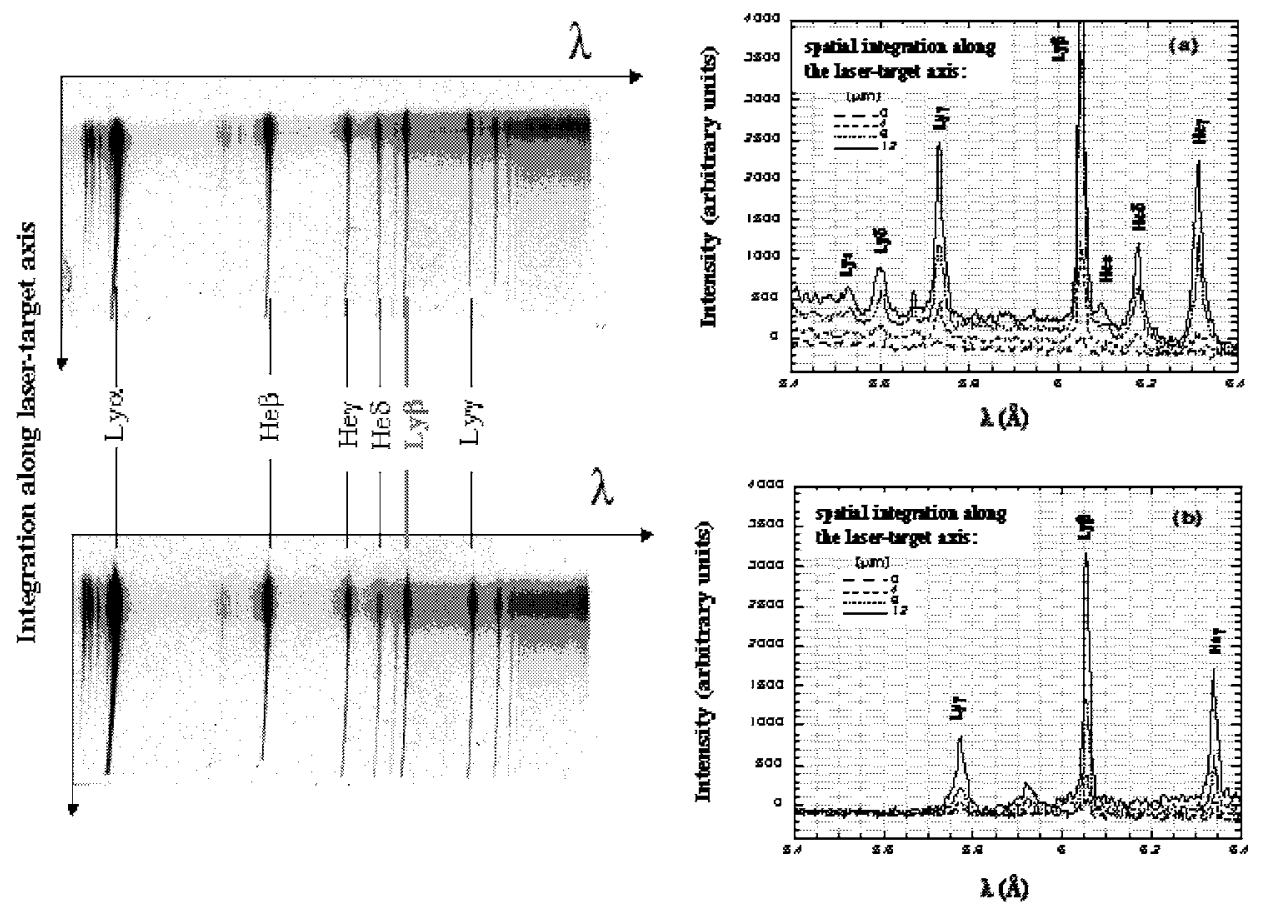

FIGURE 5. Two films and their respective corrected scans: the upper corresponds to a previous target and the lower to a newly designed target.

\section{OPACITY MEASUREMENTS BY EMISSION SPECTROSCOPY}

\section{The Experimental Protocol}

The measures presented here deal with aluminum bound-bound opacities. First, emission experiments have been modeled to extract opacities. For an aluminum thickness 21 lower than $70 \mu \mathrm{m}$, the $2 \mathrm{D}$ hydro simulations show that the density and the temperature are more or less homogeneous transversally. The reabsorbed line intensity for a slice $\mathrm{dz}$ of plasma can be written as:

$$
\mathrm{I}(z, \omega, 2 l) d z=d z D S(z)\left[1-e^{-\imath(z, \omega, 2 l)}\right]
$$

where D stand for the focal spot diameter. $S(z)$ and $\tau(z, \omega, 2 l)$, respectively the source function and the optical depth, can be expressed as follow: 


$$
S(z)=\frac{\hat{\varepsilon}(z)}{\hat{k}(z)} \text { and } \tau(z, \omega, 2 l)=\varphi(z, \omega) \hat{k}(z) 2 l
$$

where the opacity $\hat{k}(z)$, the emissivity $\hat{\varepsilon}(z)$ and the normalized line profile $\varphi(z, \omega)$ have been introduced. The aim of this work is to probe the spectrally resolved opacity $k(z, \omega)=\varphi(z, \omega) \hat{k}(z)$. Introducing its value at the maximum $\omega_{0}$ of the line, we have:

$$
k(z, \omega)=k\left(z, \omega_{0}\right) \frac{\varphi(z, \omega)}{\varphi\left(z, \omega_{0}\right)}
$$

The opacity at $\omega_{0}$ can be inferred from the ratio between the two reabsorbed intensities from two different targets having $2 \mathrm{l}_{1}$ and $2 \mathrm{l}_{2}$ as thickness

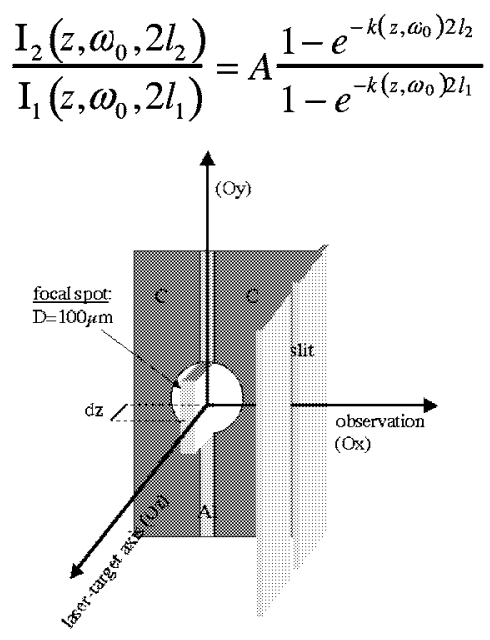

FIGURE 6. Modeling opacities and emissivities.

This ratio is written here with the assumptions that the redistribution is complete (opacity and emissivity have the same shape) and that the ionic populations are decoupled from the radiative transfer and the line profile [3]. This last point is true because the optical depth will never exceed $1(\tau \leq 1)$ for the lines in consideration. A is an adjustable parameter that should be theoretically equal to one. However it turns out to be not true from an experimental point of view. The complication here comes from the control of the position (z) and the extension (dz) of the plasma, the reproducibility of the focal spot diameter (D) and of the laser intensity for the two shots leading to the same source functions $S(z)$. All these experimental uncertainties are gathered in the A parameter, which can be inferred from the same ratio (5) applied to the line wings that are obviously considered as optically thin (i.e. $\imath<<1$ ) even for thick foils: 


$$
\frac{\mathrm{I}_{2}\left(z, \text { wings }, 2 l_{2}\right)}{\mathrm{I}_{1}\left(z, \text { wings }, 2 l_{1}\right)}=A \frac{l_{2}}{l_{1}}
$$

The line profile in formula (3) is properly measured from the spectrally resolved intensity in the line wing domain where the plasma is optically thin. For thin targets this domain is enlarged towards the line center.

In conclusion, from emission spectroscopy and using this model, the spectrally resolved opacity should be known accurately at the maximum and in the wings but an extrapolation should be done for the intermediate spectral regions. In fact as we will see, the measures for the maximum are submitted to uncertainties.

\section{Al Lyß Opacity Results and Discussion}

The figure 7 a) consists of two different opacity profiles for Al Lyß obtained from the comparison between 20 and $60 \mu \mathrm{m}$ targets for the upper one, and 20 and $40 \mu \mathrm{m}$ targets for the lower one. The extrapolations explained above are shown with dotted lines. From the described method, the opacity profiles should be comprised between these two curves. As the transverse inhomogeneity grows progressively when increasing the aluminum thickness from 40 to $60 \mu \mathrm{m}$, the error bars drawn on the graph are due to the limits of the validity of the model. We emphasize that all these opacity measurements concern the same plasma zone, i.e. the shocked zone.
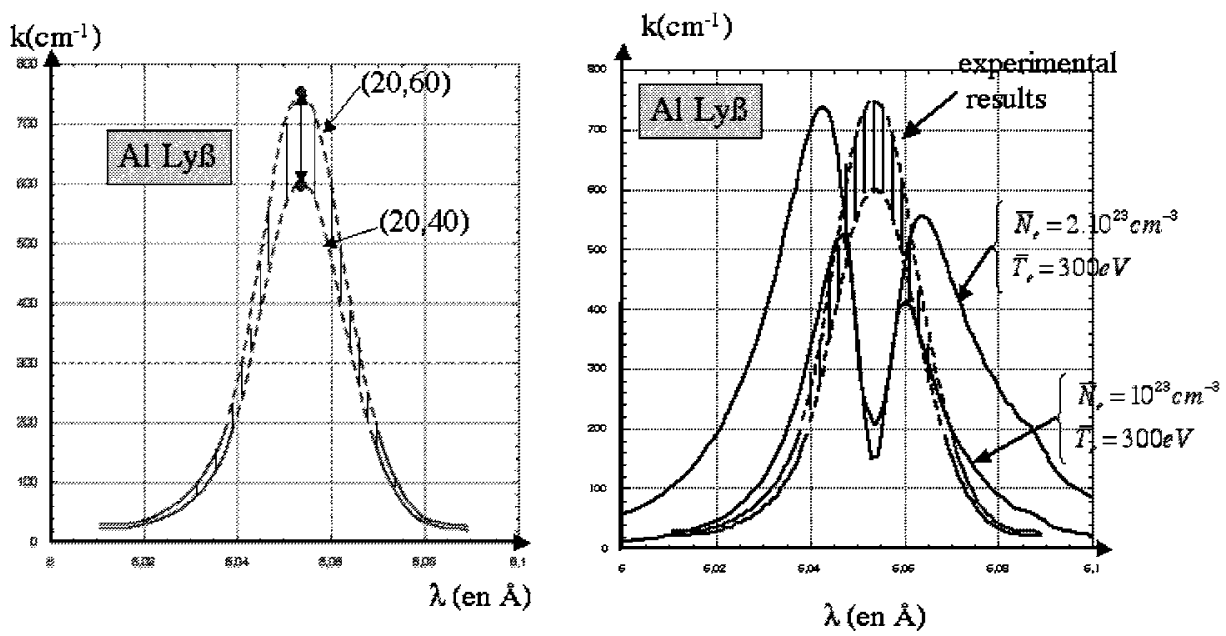

FIGURES 7. a) Al Lyß opacity profile. The upper and lower curves are obtained respectively from the comparisons between 20 and $60 \mu \mathrm{m}$ targets and 20 and $40 \mu \mathrm{m}$ targets. b) Opacity simulations and experimental data. The simulations are plotted with solid lines for the temperature $300 \mathrm{eV}$ and the densities $10^{23}$ and $2.10^{23} \mathrm{~cm}^{-3}$ respectively. The experimental estimate is in the chopped domain.

The opacity measurements have been compared to simulations [2]. The code IDEFIX, based on a quasi-molecular model, has been applied to opacities and emissivities. This code computes the line profile emitted by two nearest neighbor ions 
separated by $\mathrm{R}$ and the oscillator strengths for the same ionic separation. An average over the nearest neighbor probability distribution is performed. The codes RATION [4] or M3R [5] provide the ionic population data.

Figure $7 \mathrm{~b}$ ) presents two opacity simulations given by solid lines for the plasma conditions $\left[10^{23} \mathrm{~cm}^{-3}-300 \mathrm{ev}\right]$, and $\left[2.10^{23} \mathrm{~cm}^{-3}-300 \mathrm{eV}\right]$ respectively. The evolution with the density is drastic. On the same graph are also given the previous experimental opacity profiles (figure 7). From the comparison between the simulations and the experimental profiles, the density corresponding to the experiment is found to be $10^{23} \mathrm{~cm}^{-3}$. This density, which is a discriminating parameter, is coherent with the Inglis-Teller limit and the hydro simulations.

\section{The Hydro Simulations}

A code taking into account the radiation transport and using an Adaptative Mesh Refinement (AMR) has been adapted to our target geometry for the hydro simulations [6]. For this, the 3D geometry of the target is converted into a 2D cylindrical geometry as shown on figure 8 . For this modeling, the aluminum inner cylinder has a diameter corresponding to the thickness of the aluminum foil and the plastic outer cylinder is fitted to the focal spot.
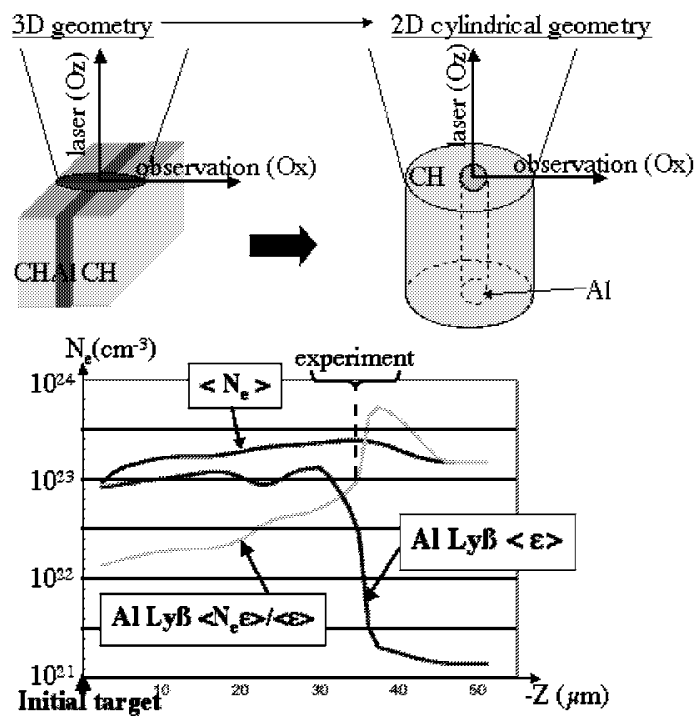

FIGURE 8. Above: cartoon of the modeling operated on the $3 \mathrm{D}$ geometry of the target before processing the $2 \mathrm{D}$ hydro simulations. Below: results of $2 \mathrm{D}$ hydro simulations. The three curves represent respectively the averaged density, the $\mathrm{Al}$ Lyß averaged emissivity and the averaged density weighed by the Al Lyß emissivity.

We have plotted on the same figure results that are time averaged and involve a transverse integration along the observation direction. The averaged density, the averaged Al Lyß emissivity, and the averaged density weighted by Al Lyß emissivity are given. The latter is the curve of interest for the purpose. According to the previous 
paragraph where the re-absorption has been investigated for the density $10^{23} \mathrm{~cm}^{-3}$, the plasma of interest corresponds to the beginning of the shock front region.

\title{
OPACITY MEASUREMENTS BY ABSORPTION SPECTROSCOPY
}

\begin{abstract}
Absorption Experiment
This last section is devoted to the description and the first results of an absorption experiment that is a more natural method for measuring opacities in cold dense plasmas. For moderately hot/cold ultra-dense plasmas characterized by moderate source functions, the natural method based on the most intense measured signal is not obvious. Moreover it is important to underline the long-term goal of our experiment that is to corroborate the previous measurements obtained from emission spectroscopy to the new ones to be obtained from absorption spectroscopy.
\end{abstract}

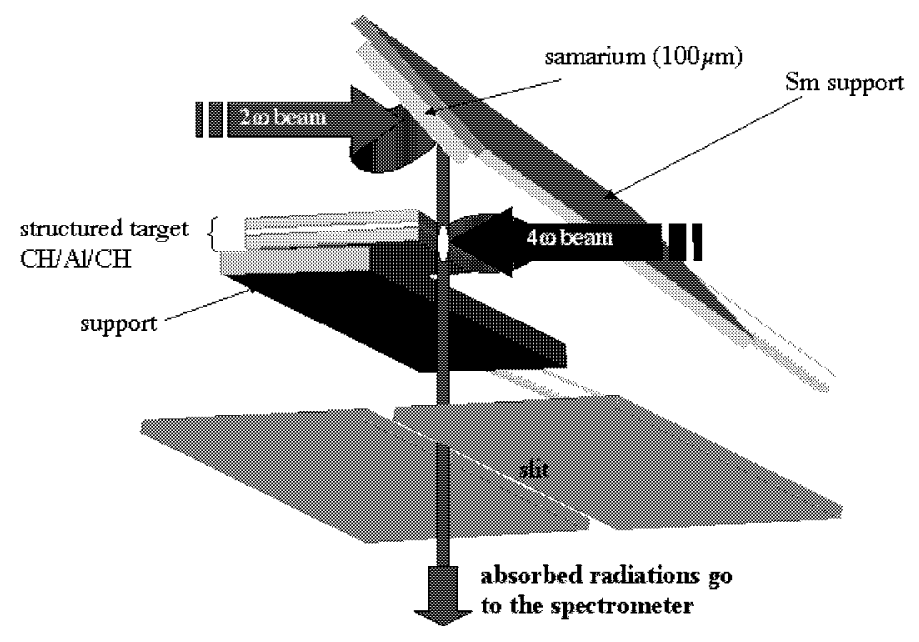

FIGURE 9. Schematic of the absorption experiment

A schematic of the experimental setup is given on figure 9. The samarium has proved to be a suitable back-lighter for the absorption through the three-layered $\mathrm{CH} / \mathrm{Al} / \mathrm{CH}$ foil described previously. The ultra-resolved transition array (UTA) exhibits a very bright quasi-continuum of emission, bright enough to observe entire absorption lines. The experimental issue is the alignment between the most emissive part of the samarium and the densest part of the aluminum plasma confined in the plastic, for the spectral window of absorption. For this purpose, the two supports of samarium and aluminum are able to move along different directions with micrometer steps. Furthermore, the thickness of the plastic on both sides of aluminum is such that the resulting plasma is optically thin. It is also very important to notice that the aluminum emission is more or less negligible with respect to the samarium emission. 


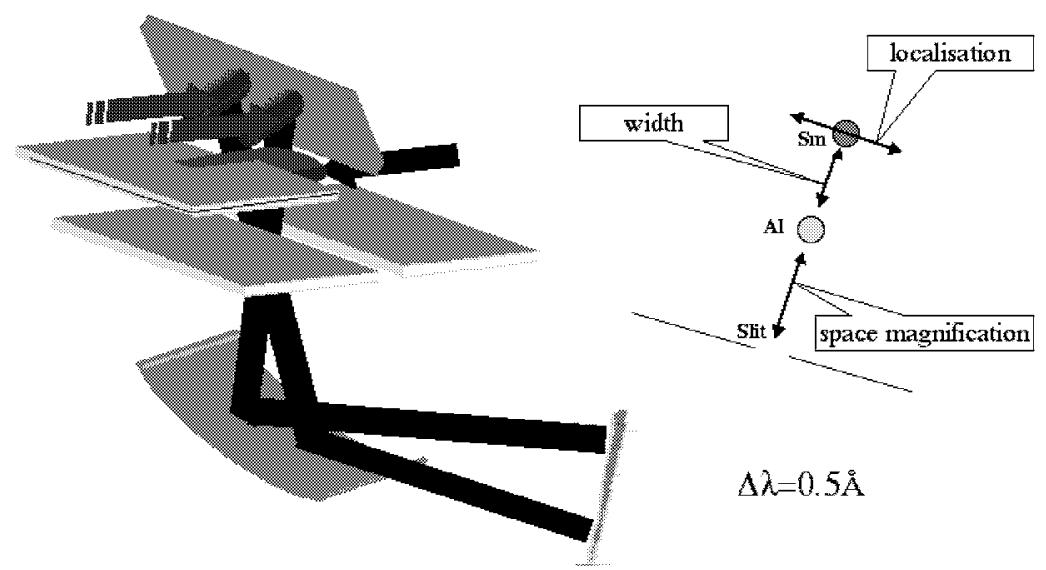

FIGURE 10. Summary of the different possible adjustments driving the magnification and the absorption spectral window.

The design of this experiment engenders typical features in the absorption spectrum. A part of this spectrum, typically a quarter, exhibits a spectral window where the samarium continuum is absorbed by the aluminum plasma. On the rest of the film, samarium and aluminum direct emissions overlap, so that both the aluminum lines and the samarium quasi-continuum can be observed. The figure 11 tries to sum up the spectrum parameters that can be adjusted along by tweaking some distances. First, the lateral position of the samarium plasma with respect to the aluminum plasma controls the localization of the spectral window where absorption takes place. On the figure, two possible focal spots are represented on the samarium with the respective paths for the absorbed radiations, resulting in two different spectral windows for the absorption spectrum. Second, the distance between the two plasmas (samarium and aluminum) is related to the width of this spectral window. The closer the samarium to the aluminum is, the larger the window is. For a separation of $1 \mathrm{~mm}$, this width is about $0.5 \AA$. Finally the separation between the aluminum plasma and the slit drives the magnification along the space integration axis, i.e. the laser-target axis.

The success of this experiment lies on the abundance of the ionic population of the lower levels involved for the aluminum transition considered. Consequently, it is easier to get absorption in Helium-like lines than Hydrogen-like lines due to the ionic populations of their respective lower levels. So the efforts were first focused on $\mathrm{Al}$ He $\gamma$ line.

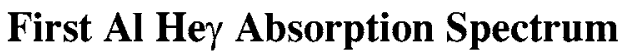

Preliminary results for the $\mathrm{Al} \mathrm{He \gamma} \mathrm{line} \mathrm{are} \mathrm{given} \mathrm{on} \mathrm{figure} \mathrm{12.} \mathrm{This} \mathrm{figure} \mathrm{gives} \mathrm{a}$ scan around this line (see the whole spectral window on figure 11) for the emission reabsorbed from the densest part of the aluminum plasma. The spectrum being absorbed through the densest part of the aluminum plasma is shown by the solid line. 
The aluminum geometrical depth was about $20 \mu \mathrm{m}$. Neglecting the aluminum selfemission, the absorbed samarium intensity is written as follows:

$$
I_{\text {absorbed-Sm }} \propto \varepsilon_{S m} e^{-\tau_{A l}}
$$

where $\varepsilon_{S m}$ stands for the emissivity and $\tau_{A l}$ for the aluminum optical depth. The aluminum optical depth at central frequency can be inferred from these considerations, and is found to be about 0.5 , leading to opacity of $255 \mathrm{~cm}^{-1}$.

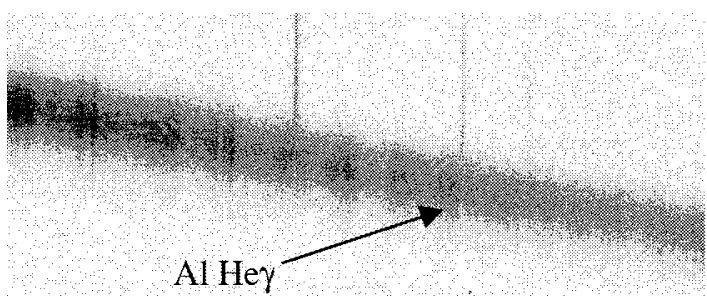

FIGURE 11. Emission collected on the film during a shot for the absorption experiment. The absorption of the Sm quasi-continuum by the Al He $\gamma$ line is pinpointed.

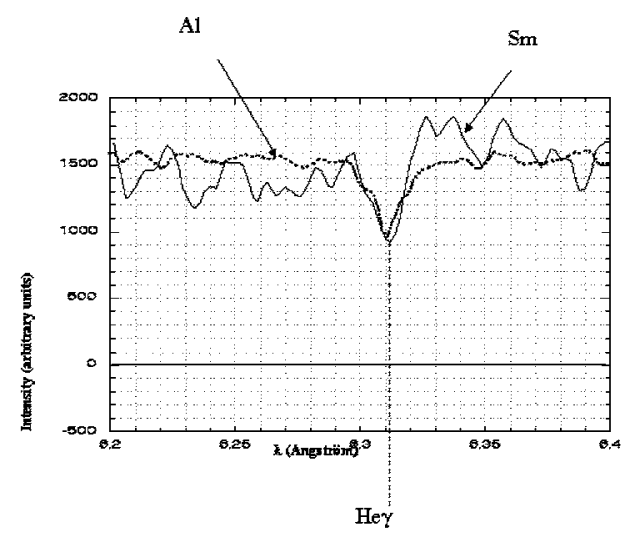

FIGURE 12. Comparison between the absorption and the emission spectra of $\mathrm{Al} \mathrm{He \gamma}$ for the same plasma conditions Solid line gives the spectrum of the samarium reabsorbed by the aluminum ultradense plasma. Dotted line corresponds to a flip of the aluminum emission for the same $20 \mu \mathrm{m}$ thickness. The zero level has been raised arbitrarily up to the samarium quasi-continuum.

On the same graph, the dotted curve reproduces the opposite of the aluminum emission spectrum, i.e. the emission of a $20 \mu \mathrm{m}$ target, for the same plasma conditions. It appears that both profiles have more or less the same shape and broadening. Actually, this isn't surprising for the optical depth is rather low.

\section{CONCLUSION}

First measures of bound-bound opacities have been performed for hot, dense laser shocked aluminum plasmas. For that, new targets and spectrographs have been 
designed and they have shown their capability for optimizing the diagnostics. We have underlined that the spectral line shape, which is the probe of ionic correlations, is determinant. We have shown the limits of emission spectroscopy that gives not a direct measure of opacity and requires the comparison between different reabsorbed emissions from plasmas resulting from different laser shots. The absorption experiment is in progress. We plan to compare emission and absorption results for selected lines (Ly $\beta, \gamma$ and $\mathrm{He} \beta, \gamma$ ) and compare systematically with opacity simulations obtained with different models in order to validate the simulations.

In the next future we plan to quantify the experimental results yet obtained for the bound-free opacities and interpret them within the frame of a model including new recombination processes due to the formation of quasi-molecules in ultra-dense plasmas.

\section{ACKNOWLEDGMENTS}

This work has been supported by the "Improve Human Potential and Socio-

Economic Base" Program of the European Community, contract n HPRI CT 19990052 .

\section{REFERENCES}

1. Gauthier, P., et al., Phys. Rev. E58, 942-950 (1998), Leboucher-Dalimier, E., et al., J. Quant. Spectrosc. Radiat. Transfer 71, $493-504$ (2001), Sauvan, P., et al., J. Quant. Spectrosc. Radiat. Transfer 71, 675-687 (2001)

2. Sauvan, P., et al., proceeding in 16th International Conference on Spectral Line Shapes (ICSLS)

3. Scott, H. A., J. Quant. Spectrosc. Radiat. Transfer 71, 689-701 (2001)

4. Lee, R. W., Whitten, B. L., and Strout, J. I., J. Quant. Spectrosc. Radiat. Transfer, 32, 91 (1984)

5. Mancini, R. C. and Minguez, E., First International NLTE Atomic Kinetic Workshop, Gaithersburg (1996)

6. Velarde, P., and Ogando, F., J. Quant. Spectrosc. Radiat. Transfer, 71, 541-550 (2001) 